\title{
THE THIRTY-FIFTH SUMMER MEETING OF THE AMERICAN MATHEMATICAL SOCIETY
}

The thirty-fifth summer meeting and thirteenth olloquium of the Society were held at the University of Colorado, Boulder, Colorado, from Tuesday to Friday, August 27-30, 1929 , preceded by the summer meeting of the Mathematical Association of America.

The four colloquium lectures by Professor R. L. Moore, each of an hour and one-quarter, were delivered on Tuesday and Thursday afternoons and Thursday and Friday mornings, with ninety-one members in attendance; a report of these lectures, by Professor G. T. Whyburn, appears in the present issue of this Bulletin. On Thursday morning the Society held sectional sessions for the reading of papers, a Section of Analysis, and a Section of Algebra. General sessions were held on Tuesday afternoon and Friday morning. The retiring address of President Snyder, entitled The problem of the cubic variety in $\mathrm{S}_{4}$, which has already appeared on pages 607-642 of the September-October issue of this Bulletin, was delivered on Thursday afternoon. In place of the joint dinner of the Society and the Association, there was an excursion to University Camp, where a dinner was served, after which there were informal speeches, Professor A. J. Kempner presiding.

This is the farthest west a summer meeting has been held since 1915, and in every particular it justified the high hopes of those interested in the expansion of the area of theSociety's activities. Boulder is picturesquely situated, the University's ample facilities were placed unreservedly and efficiently at the disposal of the visitors, the attendance was good, and the scientific standard of the meetings was high. The scientists came from twenty-eight states and Canada, representing nearly all sections of the country. Especially memorable among mathematical outings were the dinner at University 
Camp in the mountains on Tuesday evening and the day trip on Wednesday to the continental divide at Fall River Pass, nearly twelve thousand feet above sea level.

At the concluding session, by a rising vote, resolutions of thanks were passed to the President and Regents of the University, to the members of the department of mathematics, to those who furnished transportation into the mountains, and to the Boulder Country Club.

The college fraternity houses were opened for lodging the visitors, and meals were served at a cafeteria near the campus, all at modest rates.

The attendance included the following one hundred nine members of the Society:

Bacon, S. R. Benedict, William James Berry, Brink, Bruce, H. E. Buchanan, Burnam, Bussey, Cairns, C. C. Camp, A. G. Clark, Abraham Cohen, Teresa Cohen, Colyer, L. P. Copeland, C. F. Craig, Curtiss, D. R. Davis, H. T. Davis, Dean, Denton, Dines, Dodd, Edmonson, Epsteen, Erikson, H. P. Evans, Fitterer, L. R. Ford, W. B. Ford, Fry, Gaba, J. L. Gibson, Gouwens, Griffin, Griffiths, W. L. Hart, Hazard, E. R. Hedrick, Hennel, Herr, Hicks, Hildebrandt, J. C. Hughes, Hurst, C. A. Hutchinson, Ingraham, Dunham Jackson, Kempner, Kendall, Kindle, Kuhn, Latimer, D. N. Lehmer, Lester, A. J. Lewis, Lubben, McFarland, McMaster, Mickelson, W. E. Milne, U. G. Mitchell, Mossman, R. L. Moore, W. K. Nelson, Newton, Nyswander, H. L. Olson, F. W. Owens, H. B. Owens, Pierce, Pierpont, M. B. Porter, Rambo, Raynor, Rawles, Rechard, Remick, R. G. D. Richardson, Richert, H. L. Rietz, J. H. Roberts, T. G. Rodgers, Roever, Rutt, Shewhart, Sisam, Skinner, Smail, C. E. Smith, E. R. Smith, G. W. Smith, R. G. Smith, Virgil Snyder, Spooner, Eugene Stephens, Stouffer, Tappan, Tripp, Tyler, L. E. Ward, Weida, Weiss, Wester, J. J. Wheeler, M. B. White, G. T. Whyburn, Winger, Zippin.

The secretary announced the entrance of the following eighty persons to membership in the Society:

Mr. James Edward Barker, University of California;

Mr. Amos Hale Black, West Virginia University;

Miss Dorothy Stahl Brady, New York University;

Mr. Frederick James Brand, University of Washington;

Mr. Joseph Hobart Bushey, University of Michigan;

Mr. Lloyd Cline Christianson, Texas Technological College;

Miss Nancy Cole, Radcliffe College;

Mr. Hollis R. Cooley; New York University;

Mr. Prescott Durand Crout, Milwaukee, Wisconsin;

Mr. Albert Eldred Currier, Harvard University;

Mr. Edward Hutchins Cutler, Harvard University; 
Dr. Dewey Colfax Duncan, University of California;

Mr. John Dyer Elder, California Institute of Technology;

Mr. Orin James Farrell, Pennsylvania State College;

Mr. Arne Fisher, Western Union Telephone and Telegraph Company, New York City;

Miss Claire Beatrice Fisher, New York University;

Miss Elizabeth Agnes Flood, University of Montana;

Mr. David Herman Frank, George Washington High School, New York City;

Mr. Kenneth Gary Fuller, Northwestern University;

Mr. Michael Goldberg, Bureau of Ordnance, United States Navy;

Mr. Jacob Goodman, Rutgers University; Mr. Edward Kenneth Haviland, Harvard University;

Mr. William Jonathan Hazard, University of Colorado;

Mr. Charles Harold Helliwell, New York University;

Mr. Magnus Rudolph Hestenes, University of Nebraska;

Mr. John James Luett Hinrichsen, Iowa State College;

Miss Catherine Hood, Colorado College;

Professor Borden P. Hoover, Carnegie Institute of Technology;

Professor Frederick W. John, New York University;

Professor Francis Edgar Johnston, George Washington University;

Dr. Franklin Wesley Kokomoor, University of Florida;

Professor Michael Kovalenko, Swarthmore College;

Mr. Lonnie Langston, Texas Technological College;

Professor Victor F. Lenzen, University of California;

Mr. Arthur James Lewis, University of Denver;

Mr. Chin-Nien Liu, Nan Kai University, Tienstin, China;

Professor Hilton Frank Lusk, College of the Pacific;

Dr. Albert Joseph McConnell, Trinity College, Dublin, Ireland;

Miss Dorothy McCoy, State University of Iowa;

Mr William Milton Maiden, New York University;

Mr. Monroe Harnish Martin, Johns Hopkins University;

Professor Wendell Earle Mason, University of California at Los Angeles;

Mr. Frederic Howell Miller, Columbia University;

Dr. Alfred King Mitchell, Johns Hopkins University;

Mr. Morris Monsky, Mutual Life Insurance Company, New York City;

Dr. Walter Lee Moore, University of Illinois;

Mr. William Richard Morgans, California Institute of Technology;

Dr. William Kelso Morrill, Johns Hopkins University;

Mr. David Moskovitz, Carnegie Institute of Technology;

Mr. J. Herbert Moss, New York University;

Professor Walter Kenneth Nelson, University of Colorado;

Mr. James Edward O'Connell, Morris Preparatory School, Little Rock, Arkansas;

Professor Edwin Glenn Olds, Carnegie Institute of Technology;

Miss Emma Julia Olson, Northwestern University;

Mr. Charles Kenneth Payne, New York University;

Dr. Joseph William Peters, University of Illinois; 
Professor Saul Pollock, Coast Artillery Corps, Fort Collins, Colorado;

Mr. Griffith Baley Price, Harvard University;

Professor A. W. Richeson, University of Maryland;

Professor Ramon Salas-Edwards, University of Chile, Santiago, Chile;

Mr. William Francis Shaw, vice-president, American Rio Grande Land and Irrigation Company;

Miss C. Grace Shover, Ohio State University;

Mr. Clifford Edward Smith, Fresno State College;

Professor Turner L. Smith, Carnegie Institute of Technology;

Mr. Clyde Allen Spicer, Johns Hopkins University;

Dr. Lee Houghton Swinford, University of California;

Professor Herbert Tate, McGill University;

Mr. Henry Peter Thielman, Ohio State University;

Mr. William Rae Thompson, Yale University;

Professor Arthur Tilley, New York University;

Professor Louis Russell Weber, Friend's University, Wichita;

Mr. Edward Henry Wells, Princeton University;

Frofessor George Warren White, College of the Pacific, Stockton, California;

Professor Edwin A. Whitman, Carnegie Institute of Technology;

Mr. Hassler Whitney, Yale University;

Mr. Charles Robert Wilson, Rutgers University;

Mr. Herbert Paul Wirth, College of the City of New York;

Mr. Kamcheung Woo, University of California;

Professor Leon P. Woods, Northeastern State Teachers College, Tahlequah, Oklahoma;

Dr. Minoru Yoshida, Sendai, Japan.

Eight applications for membership were received.

The Secretary also announced the following election to sustaining membership:

Members of the Department of Mathematics in Brown University.

The following appointments were announced: to represent the Society at the inauguration of President Clarence A. Barbour at Brown University, Professor G. D. Birkhoff; to represent the Society at the fiftieth anniversary celebration of the American Society of Mechanical Engineers, Dr. G. A. Campbell and Professor E. V. Huntington; as Committee on Arrangements for the summer meeting of 1930, at Brown University, Professors C. R. Adams (chairman), J. L. Coolidge, C. H. Currier, R. G. D. Richardson and $\mathrm{H}$. W. Tyler.

It was announced that for 1929-30 the National Academy of Sciences had allotted to the Society from funds donated 
by the General Education Board the sum of $\$ 4500$ to be distributed as follows: Bulletin, $\$ 500$; Transactions, $\$ 1500$; American Journal of Mathematics, \$1500; Colloquium, $\$ 1000$.

The list of nominations for officers and other members of the Council was adopted and ordered printed on the ballot.

Titles and abstracts of the papers read at this meeting follow below. The papers numbered 1 to 7 were read at the general session on Tuesday afternoon, President Hedrick presiding; the papers numbered 8 to 23 were read before the Section of Analysis, ex-President Snyder and Professor Curtiss presiding; those numbered 24 to 43 before the Section of Algebra, Vice-President Rietz and Professor Cohen presiding; those numbered 44 to 60 at the general session on Friday morning, President Hedrick and Professor Tyler presiding. Dr. W. D. Baten was introduced by Professor J. A. Shohat and Professor Uspensky by the Secretary. The papers of Ayres, Campbell, Carson, Garver, Hill, Kempner, Langer, LaPaz, Lehmer, Lubben, McDonnell, Marden, Poritsky, Rainich, Robinson, Roth, Rowe, Rutledge, Simmons, Thielman, Vandiver, Ward (second paper), Wilder and Wilson were read by title.

1. Professor J. A. Nyswander: On the solutions of linear differential equations.

This paper gives a simple and direct derivation of the form of the solution of a general system of linear differential equations in the vicinity of a point $t=0$ which is an isolated uniform singularity for the coefficients of the system of differential equations. Solutions of the system in the vicinity of a regular point $t=t_{0}$ are determined so that after a circuit about the point $t=0$, certain specified changes occur in the solutions. In order that these changes shall result from analytic continuation about the singular point $t=0$ certain relations among the coefficients of the solutions must exist. That these relations can be satisfied is proved by differentiating a set of identities obtained from the characteristic determinant. The form of the solutions is established under entirely general hypotheses regarding the roots of the characteristic equation, without recourse to the theory of transformations.

2. Professor C. H. Sisam: On three-dimensional varieties with six generations by right lines. 
In this paper, the author shows that the varieties mentioned in the title are cubic hypersurfaces in space of four dimensions.

\section{Professor G. Y. Rainich: Decomposition of a symmetric tensor in space-time.}

It is proved in this paper that in four-dimensional pseudo-euclidean space a non-singular symmetric tensor of rank two can be split up in only one way into two parts which possess respectively the formal properties of the hydrodynamical tensor (tensor of matter) and the electromagnetic stress-energy tensor. Applying this result and results previously established to the contracted Riemann tensor, it follows that curvature of space-time determines uniquely the field of matter as well as the electromagnetic field. In the singular case the decomposition is not unique: one of the tensors (each of which represents in a sense radiation) can be chosen to a certain degree arbitrarily.

4. Professor L. L. Dines: A mininum problem from physiology.

The sensitive portion of the retina consists of a collection of circular surfaces ("cone" surfaces) of equal known radii, uniformly distributed, the intervening area being non-sensitive. Assuming known the size of the smallest circular image which can be seen, it is desired to know what is the minimum area of cone surface which such an image can occupy. This question, proposed by a colleague of the author, is considered in the present note.

5. Professor E. L. Dodd: Probability as expressed by asymptotic limits of pencils of sequences.

In order to deal with probability without recourse to the much criticized notion of "cases equally likely," a set of sequences is introduced, called a pencil of sequences, inasmuch as they radiate (figuratively speaking) from a common origin. If $H$ means "heads," and $T$ means "tails," an individual sequence, HHTH $\cdots$ is not conceived as giving rise to any limit. But for a pencil an actual limit is involved when only an evanescent percentage of the sequences fail to conform to certain inequalities. An exact and rather simple formulation - avoiding all non-mathematical phrases and conceptions, indeed, avoiding the word "probability" itself-is given to the Bernoulli theorem, its extensions, and its inverse. This involves only the counting of sequences, and the use of ordinary limits. This may give the student of probability a clearer conception of the Bernoulli inverse than he gets from the following statement: With arbitrary $a$ priori probabilities, there is ultimately a very high $a$ posteriori probability that the empirical probability will closely approximate that unknown probability that gives rise to the productive probability. References are made to the work of Cantelli, von Mises, Borel, Lomnicki, and Steinhaus.

6. Professor W. E. Milne: On the degree of convergence of expansions on an infinite interval. 
The author studies the rate of convergence of expansions in terms of the characteristic functions which are associated with the differential equation $d^{2} u / d x^{2}+(\lambda-q(x)) u=0$ and which are also subject to the conditions $\lim _{x= \pm \infty} u=\lim _{x= \pm \infty} u^{\prime}=0$. With appropriate restrictions of the function $q(x)$ an approximate formula is obtained relating the $n$th characteristic number $\lambda_{n}$ with $n$. In particular it is found that the order of magnitude of $\lambda_{n}$ is always less than $n^{2}$. It is shown that if $f(x)$ has a continuous $k$ th derivative of bounded variation in the infinite interval, and satisfies certain conditions at infinity, then the remainder after $n$ terms of the expansion of $f(x)$ is $O\left(\lambda^{-k / 2}\right)$ in any fixed finite interval.

7. Professor H. T. Davis: Properties of the Laurent expansion of differential operators of infinite order with constant coefficients.

This paper exhibits some simple properties of the development of the symbolic operator $G(z)=1 / F(z)$, in which we abbreviate $F(z)=a_{0}+a_{1} z+a_{2} z^{2}$ $+\cdots$, where $z=d / d x$, in annuli formed by concentric circles about the origin. The following theorem is proved: If $H(z)$ designates a Laurent expansion of $G(z)$ in an annulus formed by two concentric circles about the origin and if $G(z)$ is any other expansion about the origin, then the function $U(x)=\{H(z)-G(z)\} \rightarrow f(x)$, where $f(x)$ is arbitrary to within the limits of the existence of the right hand member, is a solution of the homogeneous equation, $F(z) \rightarrow u(x)=0$. The case where $G(z)$ is a rational function is shown to yield the fundamental operator of the Heaviside operational calculus when the expansion is that in the region exterior to all the poles of the function.

8. Dr. W. D. Baten: The evaluation of certain definite integrals by the use of probability functions.

The object of this paper is to present three methods of evaluating certain definite integrals by using probability functions. The first method consists in finding the probability law or function for the sum of $n$ independent variables, which are each subject to given probability laws, by Mayr's method and comparing this with the probability for the same sum obtained by Dodd's method. Method two consists in finding the probability law for $n$ independent variables, which is expressed as an integral, and then allowing $n$ to be equal to 1 . Method three is founded on setting up a probability function containing the integral to be evaluated as a factor, determining a second probability function defined over the same interval, and then finding a third probability function for a chosen relation of the variables under consideration. The integral need not be a factor of the first function, but should arise somewhere in the process. The first two methods, as far as the writer knows, seem to be new.

9. Professor D. R. Davis: Integrals whose extremals are a 2n-parameter family of curves.

One considers here a given $2 n$-parameter family of arcs of the form 
$y_{i}=y_{i}\left(x, a_{1}, \cdots, a_{n}, b_{1}, \cdots, b_{n}\right)(i=1, \cdots, n)$, and seeks the properties which are sufficient to ensure that they are the extremal arcs of an integral of the form $I=\int_{x}^{x_{2}} f\left(x, y_{1}, \cdots, y_{n}, y_{1}{ }^{\prime}, \cdots, y_{n}{ }^{\prime}\right) d x$. These properties are determined under the hypothesis that the differential equations $y_{i}{ }^{\prime \prime}$ $=F_{i}\left(x, y_{1}, \cdots, y_{n}, y_{1}{ }^{\prime}, \cdots, y_{n}{ }^{\prime}\right)$, found by eliminating the $2 n$ parameters $a_{1}, \cdots, a_{n}, b_{1}, \cdots, b_{n}$, are the solutions for $y_{i}{ }^{\prime \prime}$ of a system of equations of the form $H_{i}\left(x, y_{1}, \cdots, y_{n}, y_{1}{ }^{\prime}, \cdots, y_{n}{ }^{\prime}, y_{1}{ }^{\prime \prime}, \cdots, y_{n}{ }^{\prime \prime}\right)=0$, whose equations of variation are self-adjoint. The general type of integral whose extremals are the $\operatorname{arcs} y_{i}=a_{i} x+b_{i}(i=1, \cdots, n)$ is completely determined.

10. Professor D. R. Davis: Determining integrals whose extremals are parabolas.

In this paper the inverse problem for the trajectory of a particle under the action of gravity is considered. Properties of its characteristic differential equations are developed and a general type of solution is obtained. From the latter the integrals desired are completely determined,

\section{Professor W. L. Hart: Infinite systems of non-linear equations and related integral equations.}

Let $x$ and $y$ represent points in real Hilbert space of infinitely many dimensions. Without employing convergent infinite determinants, the author establishes the unique existence of a solution $x(y)$ of the following infinite system of equations (1): $f_{1}(x, y)=0\left[x \equiv\left(x_{1}, x_{2}, \cdots\right)\right.$; $\left.y \equiv\left(y_{1}, y_{2}, \cdots\right) ; i_{1} 1,2, \cdots\right]$ in a neighborhood of a solution $(x=a$, $y=b)$. The most important of the hypotheses used are that the matrix $F \equiv(\partial f(x, y) / \partial x)$ is limited in the Hilbert sense, that $F(a, b)$ possesses a unique limited reciprocal, and that $[F(x, y)-F(a, b)]$ possesses a bound $g(x, y)$, continuous at $(a, b)$, where $g(a, b)=0$. By use of the properties of limited multilinear forms, it is demonstrated that the results for (1) can be applied in the case of various infinite systems of non-linear equations in infinitely many unknowns. Each non-linear system considered can be used to establish the existence of continuous solutions of a certain non-linear integral equation. The transition from an integral equation to a system of the form (1) is made by the usual method which introduces the Fourier constants of the continuous functions involved relative to some complete orthogonal system.

12. Mr. C. M. Erikson: A direct solution of systems of homogeneous linear difference equations having constant coefficients.

The paper gives in detail the method and theory of obtaining the solutions of simultaneous systems of linear difference equations with constant coefficients. The first case treated is the simple one in which the characteristic equation, $D(\lambda)=0$, has distinct roots. The solutions are expressed in terms of first minors of the characteristic determinant $D\left(\lambda_{i}\right)$. The next case considered is the one in which $D(\lambda)=0$ has a triple root. The most general case is then treated, under hypotheses that include every 
special case that could arise. In this case the solutions are obtained corresponding to a multiple root $\lambda=\lambda_{i}$ of the characteristic equation $D(\lambda)=0$, where entirely general assumptions are made regarding the vanishing of minors of different orders in the characteristic determinant $D\left(\lambda_{i}\right)$. The solutions thus obtained are shown to form a fundamental set. The problem is treated without making use of the theory of transformations.

\section{Professor C. C. Camp: On multiparameter expansions} associated with a differential system and auxiliary conditions at several points in each variable.

For $p=2$ the system $d X_{i} / d x_{i}+\sum_{j=1}^{p} \lambda_{j} a_{i j}\left(x_{i}\right) X_{i}=0,(i=1,2, \cdots, p)$, and boundary conditions of the Wilder type in each lead to suitable characteristic solutions provided the $a_{i j}$ are properly restricted. It is sufficient if $a_{i j}\left(x_{i}\right)$ is a real, integrable function, either identically zero or of constant sign, which maintains its mean value in each segment of the boundary interval $a_{i} \leqq x_{i} \leqq b_{i}$, and the determinant $\left|\int_{a_{i}}^{b_{i}} a_{i j} d x_{i}\right| \neq 0$. If the $k_{i}$ segments have lengths commensurable with $b_{i}-a_{i}$ and are the same for $i=1,2$, the factors containing one parameter may be eliminated by Sylvester's method, so that the characteristic values of the other will be zeros of an entire function. For $k_{i}>3$ the restrictions on $a_{i j}\left(x_{i}\right)$ may be lightened in the interior subintervals.

14. Dr. Lincoln LaPaz: Problems of the calculus of variations with prescribed transversality conditions.

The transversality condition for a problem of minimizing the integral

is

$$
\begin{aligned}
& I=\int_{x_{1}}^{x_{2}} f\left(x, y_{1}, \cdots, y_{n}, y_{1 n}^{\prime}, \cdots, y_{n}^{\prime}\right) d x \\
& \left(f-y_{\mu}^{\prime} \partial f / \partial y_{\mu}^{\prime}\right) d x+\partial f / \partial y_{\mu}^{\prime} d y_{\mu}=0
\end{aligned}
$$

( $\mu$ is umbral, ranging from 1 to $n$ ). In this paper it is shown that a relation (3) $d x+T_{\mu}\left(x, y_{1}, \cdots, y_{n}, y_{1}^{\prime}, \cdots, y_{n}^{\prime}\right) d y_{\mu}^{\prime}=0, \quad 1+y_{\mu}^{\prime} T_{\mu} \neq 0$,

can be the transversality relation (2) of a problem (1) only in case the $T_{i}$ are such that a certain system of $n$ Iinear homogeneous partial differential equations is a complete system. Necessary and sufficient conditions for this system to be complete are that

$$
y_{\mu}^{\prime}\left(T_{m} \partial T_{k} / \partial y_{\mu}^{\prime}-T_{k} \partial T_{m} / \partial y_{\mu}^{\prime}\right)+\left(\partial T_{k} / \partial y_{m}^{\prime}-\partial T_{m} / \partial y_{k}^{\prime}\right)=0,
$$

$(m, k=1, \cdots, n, m<k)$. For $n$ functions $T_{i}$ satisfying (4) the most general integral (1) for which (3) is the transversality condition is determined. The results obtained supplement those found by a different method by Rawles (Transactions of this Society, vol. 30 (1928), pp. 765-784).

15. Dr. Lincoln LaPaz: An inverse problem of the calculus of variations for multiple integrals.

The Lagrange equation of a problem of minimizing an $n$-tuple integral $J=\int_{(n)} f\left(x_{1}, \cdots, x_{n}, z, p_{1}, \cdots, p_{n}\right) d x_{1} \cdots d x_{n},\left(p_{i}=\partial z / \partial x_{i}\right)$, must be of the 
form $A_{\mu \nu}\left(x_{1}, \cdots, x_{n}, z, p_{1}, \cdots, p_{n}\right) p_{\mu \nu}+B\left(x_{1}, \cdots, x_{n}, z, p_{1}, \cdots, p_{n}\right)=0$, $\left(p_{i j}=\partial^{2} z / \partial x_{i} \partial x_{j}, A_{i j}=A_{j i}, i, j, \mu, \nu=1, \cdots, n, \mu\right.$ and $\nu$ umbral), with an equation of variation which is self-adjoint on every hypersurface $z=z\left(x_{1}, \cdots, x_{n}\right)$. It is shown that if an equation $F\left(x_{1}, \cdots, x_{n}, z, p_{1}, \cdots\right.$, $\left.p_{n}, p_{11}, \cdots, p_{n n}\right)=0$ has the above properties, there is always an integral $J$ having $F=0$ as its Lagrange equation. The most general such integral has an integrand function of the form $f=C\left(x_{1}, \cdots, x_{n}, z, p_{1}, \cdots, p_{n}\right)$ $+D\left(x_{1}, \cdots, x_{n}, z\right)+E_{\mu}\left(x_{1}, \cdots, x_{n}, z\right) p_{\mu}+\partial w_{\mu} / \partial x_{\mu}$, where $C$ is a particular solution of the system $\partial^{2} C / \partial p_{i} \partial p_{j}=A_{i j}$, and $\left(D, E_{i}\right)$ is a particular solution of $\partial D / \partial z-\partial E_{\mu} / \partial x_{\mu}=\partial^{2} C / \partial p_{\mu} \partial x_{\mu}+\left(\partial^{2} C / \partial p_{\mu} \partial z\right) p_{\mu}-\partial C / \partial z-B$, and where the $w_{i}$ occurring in the sum of total derivatives with respect to $x_{i}$ are arbitrary functions of $x_{1}, \cdots, x_{n}, z$ alone. The method of proof is similar to that used by Davis (Transactions of this Society, vol. 30 (1928), pp. 710-736) in connection with an analogous question for simple integrals $I=\int_{x_{1}}^{x_{2}} f\left(x, y, z, y^{\prime}, z^{\prime}\right) d x$.

16. Mr. H. P. Thielman: The application of fractional operations to a class of integral equations occurring in physics.

This paper is concerned with the theory of a class of Volterra integral equations of the first kind occurring in physics. The methods used are those of fractional operators and Volterra's calculus of composition. The theorems and results of Wrinch and Nicholson (Philosophical Magazine, (7), vol. 4(1927), pp. 531-560) are obtained as special cases of the author's more general and elegant theory.

17. Mr. L. B. Robinson: On a lacuna in the theory of systems of partial differential equations.

In his book Les Systèmes d'Equations aux Dérivées Partielles, Riquier examines in detail the passivity conditions of such systems, but does not consider the case where the passivity conditions entrain relations among the independent variables. The author has partly succeeded in filling in this lacuna. Consider the three equations

(1) $\quad \partial u / \partial x=f(x, y, z, u), \partial u / \partial y=\phi(x, y, z, u), \partial u / \partial z=\psi(x, y, z, u)$.

This case illustrates that for $n$ equations. Supposing certain relations between the independent variables

$$
\begin{aligned}
& \partial u / \partial x=f(x, \eta(x), \zeta(x) ; u), \\
& \partial u / \partial y=\phi\left(\eta-1(y), y, \zeta\left[\eta_{-1}(y)\right] ; u\right), \\
& \partial u / \partial z=\psi\left(\zeta_{-1}(z), \eta\left[\zeta_{-1}(z)\right], z ; u\right),
\end{aligned}
$$

where $\eta$ and $\eta_{-1}$ and $\zeta$ and $\zeta_{-1}$ are pairs of inverse functions such that

$$
\begin{aligned}
& \eta_{-1}(y) d y=x \eta^{\prime}(x) d x, \zeta_{-1}(z) d z=x \zeta^{\prime}(x) d x, \\
& \eta\left[\zeta_{-1}(z)\right] d z=\eta(x) \zeta^{\prime}(x) d x, \quad \zeta\left[\eta_{-1}(y)\right] d y=\zeta(x) \eta^{\prime}(x) d x,
\end{aligned}
$$

$u$ can be computed in terms of $x$ and definite integrals involving $\eta(x)$ and $\zeta(x)$, or in terms of $y$ and definite integrals involving $\eta_{-1}(y)$ and $\zeta\left[\eta_{-1}(y)\right]$, or in terms of $z$ and definite integrals involving $\zeta_{-1}(z)$ and $\eta\left[\zeta_{-1}(z)\right]$, the definite integrals arising in the process of solving system (2) by successive approximations. Now keeping in mind the equalities (A), we find $\eta(x)$ 
and $\zeta(x)$ by forming two integro-differential equations which can easily be solved, as the kernals do not depend on the upper limits of integration. This method can be extended to systems of equations of the second order.

18. Professor George Rutledge: The basic power series of interpolation.

The coefficients of the polynomial $P^{(2 n)}(x)$, determined by the values $f(-n), \cdots, f(0), \cdots, f(n)$, are expressed in the form of series the terms of which are independent of $n$. Conditions for convergence of $P^{(2 n)}(x)$ are readily derived from this form of expansion of the coefficients. This paper is a direct continuation of the paper of similar title published in the Transactions of this Society (vol. 26 (1924), p. 113).

19. Dr. Hillel Poritsky (National Research Fellow): On certain polynomial approximations to analytic functions of a complex variable.

This paper deals with the convergence to $f(z)$ of certain polynomials $P_{m, n}(z)$ as $n$ becomes infinite, where $P_{m, n}(z)$ is of degree $m n-1$ at most and is uniquely determined by $m n$ conditions requiring that its derivatives of order $0, m, \cdots, m(n-1)$ at $m$ given fixed points $a_{1}, a_{2}, \cdots$, $a_{m}$, no two of which are alike, be equal respectively to the same order derivatives of $f(z)$ at the same points. The case $m=1$ leads essentially to Taylor's expansion of $f(z)$ about $z=a_{1}$, but is not typical of the general case $m>1$. Thus, mere analyticity of $f(z)$ in any region will not suffice to ensure the convergence of $P_{2, n}(z)$ to $f(z)$ as $n$ becomes infinite. This may be seen by putting $a_{1}=0, a_{2}=\pi$, and choosing $f(z)=\sin z ; P_{2, n}(z)$ will then identically vanish. Sufficient conditions for convergence of $P_{m, n}(z)$ to $f(z)$ as $n$ becomes infinite, it is shown, are that $f(z)$, in addition to being an integral function of $z$, satisfy an inequality $|f(z)|<\mathrm{Ce}^{k|z|}, k<K$, where $C, k$ are constants, and $K$ is a constant determined by $a_{1}, a_{2}, \cdots, a_{m}$; the convergence then takes place for all $z$. For $m=2, K=\pi /\left|a_{1}-a_{2}\right|$. In the case $m=2$ necessary conditions that are nearly sufficient are also found.

20. Dr. Morris Marden (National Research Fellow): On the zeros of solutions of a generalized Laplacian differential equation.

This article aims to furnish specific means for describing qualitatively the distribution of the zeros of any solution of the equation

$$
\left.d^{2} U / d z^{2}+(a+b / z)(d U / d z)+(c+d / z)+f / z^{2}\right) U=0
$$

where $z$ is a complex variable, but $a, b, c, d, f$ are real constants. These means are constructed as follows: Through the transformation

$$
U=z^{-b / 2} e^{-a z / 2} w,
$$

equation (1) is converted into one of the form

$$
d^{2} w / d z^{2}+\left(A+B / z+C / z^{2}\right) w=0 .
$$

Every solution of the new equation has, with the possible exception of the 
point $z=0$, the same zeros as the corresponding solution of (2). Now by use of Hille's method of "zero-free regions" (Arkiv för Matematik, vol. 18 (1924), pp. 1-56) information can be gathered which, with a few moments' additional work, enables one to locate regions where neither any particular solution of (2) nor its derivative vanishes. The efficacy of the so-constructed means is illustrated by the study of solutions of (2) with the property $W(0)=0$ or $W(+\infty)=0$, where $W(z)=\overline{w(z)} w^{\prime}(z)$.

21. Professor R. E. Langer: A class of irregular boundary problems of the second order.

The characteristic parameter values for the boundary problem of an ordinary linear differential equation are determined as the roots of a relation (1): $\sum\left[\alpha_{i}\right] e^{\rho \beta_{i}}=0$, in which $\rho$ is the parameter, the $\beta_{i}$ are complex constants, and $\left[\alpha_{i}\right]=\alpha_{i}+\alpha_{i 1} / \rho+\cdots$. With this transcendental equation there is associated in the complex plane the convex polygon which has vertices only at points of the set $\beta_{i}$ and which contains all the points $\beta_{i}$ in its in. terior or on its perimeter. The boundary problem is called "regular" if in equation (1) $\alpha_{i} \neq 0$ for those values of $i$ for which $\beta_{i}$ is a vertex of the polygon. Only for particular differential equations has the irregular boundary problem been studied. The present paper considers the problem of the general differential equation of the second order for the irregular cases in which the quantities $\left[\alpha_{i}\right]$ vanish to various degrees in $\rho$ but not identically. It is shown that the associated expansions of arbitrary functions are in these cases summable by suitable means under hypotheses similar to those which ensure convergence of the expansions in the regular cases.

22. Professor L.E. Ward: On the differentiation and integration of certain formal series.

The series considered are those associated with the differential system $u^{\prime \prime \prime}+\rho^{3} u=0, u(0)=u^{\prime}(0)=u(\pi)=0$. Necessary and sufficient conditions that the third derived series of the formal series for a function capable of being expanded in a series of the characteristic functions of this differential system should be the formal series for the third derivative of the function are obtained. It is proved that the threefold term-by-term indefinite integration of the formal series for a function yields the formal series for a certain function, and a formula for the latter is found.

23. Professor L. E. Ward: On the determination of the formal coefficients in a certain expansion problem.

If the functions $u_{k}(x)$ are the characteristic functions of the differential system $u^{\prime \prime \prime}+\rho^{3} u=0, u(0)=u^{\prime}(0)=u(\pi)=0$, and $f(x)$ is a function capable of expansion in a series of these characteristic functions, and if $R_{n}(x)=f(x)-\sum_{k=1}^{n} a_{k} u_{k}(x)$, it is shown that $\int_{0}^{\pi} R_{n}(x) R_{n}(\pi-x) d x$ has an extremum when the $a_{k}$ 's are the coefficients of the formal series for $f(x)$.

24. Professor J. V. Uspensky: On a new method for finding units in cubic rings of a negative discriminant.

The basic idea of this new method can be best explained for the simplest 
case of a cubic ring $\left[1, \theta, \theta^{2}\right]$ where $\theta$ denotes a real root of the equation $\theta^{3}=a$. Set $\phi=x+y \theta+z \theta^{2}, \phi=\phi^{\prime} \phi^{\prime \prime}, \phi^{\prime}$ and $\phi^{\prime \prime}$ being conjugate forms to $\phi$, and similarly $\omega=Z+Y \theta+Z \theta^{2}, \Omega=\omega^{\prime} \omega^{\prime \prime}$. Following Hermite's suggestion we introduce the ternary quadratic form $f=2 \phi+\phi^{2} / \Delta$ containing a variable positive parameter $\Delta$, whose adjoint form differs from $F=\omega^{2}$ $+2 \Omega / \Delta$ by an irrelevant numerical factor. But instead of considering successive minima of the form $f$ for a continuously increasing parameter, as Hermite suggests, it seems more advantageous to consider systems of integers $x, y, z ; X, Y, Z$ satisfying for every $\Delta$ the conditions $f \leqq 4\left(a^{2} / \Delta\right)^{1 / 3}$, $F \leqq 4\left(a^{2} / \Delta^{2}\right)^{1 / 3}, X x+Y y+Z z=0$. It can be shown that, starting with $x=1, y=0, z=0 ; X=0, Y=0, Z=1$ for $\Delta=1$ we can reach systems $x, y$, $z ; X, Y, Z$ such that either $\phi=x+y \theta+z \theta^{2}$ or $\omega=Z+Y \theta+X \theta^{2}$ will be a fundamental unit. When applied to numerical examples the new method seems to be quite satisfactory and not inferior to any known method that can be used for the same purpose.

\section{Professor H. S. Vandiver: On power characters of singular integers in a properly irregular cyclotomic field.}

A properly irregular cyclotomic field is a field defined by $\zeta=e^{2 \pi i / l}$, $l$ an odd prime, which is irregular and such that certain units $E_{n}, n=1$, $2, \cdots,(l-3) / 2$ (defined as by the writer, Proceedings of the National Academy of Sciences, vol. 15 (1929), p. 202) are not $l$ th powers of units in $k(\zeta)$. In the present paper the author shows how to determine in algebraic form the $l$ th power characters of any singular integer in a properly irregular cyclotomic field $k(\zeta)$, with respect to any ideal in this field prime to $l$. Heretofore this determination has only been made for the cases where the ideals belonged to an exponent prime to $l$. The paper will appear in the Transactions of this Society.

26. Professor H. S. Vandiver: On the composition of the class-group of ideals in an irregular cyclotomic field.

If the class number $h$ of an irregular cyclotomic field is expressed in the form $l^{a} g$, where $g$ is prime to $l$ and the cylotomic field is defined by $e^{2 \pi i / l}$, with $l$ an odd prime, then the $g$ th powers of all ideals in the field may be separated into classes which form an abelian group under multiplication. In the present paper the invariants of this group of classes are shown to depend on the divisibility of certain Bernoulli numbers by powers of $l$, in the case where the cyclotomic field is properly irregular. Properties of a basis for this group are also given. The investigation gives a number of other results concerning the class-field of a cyclotomic field and a basis for the singular primary numbers in the field. The paper will appear in the Transactions of this Society.

27. Professor H. S. Vandiver: Some properties of a certain system of independent units in a cyclotomic field.

Pollaczek (Mathematische Zeitschrift, vol. 21 (1924), pp. 1-38) proved 
that in the field $k(\zeta), \zeta=e^{2 \pi i / l}, l$ an odd prime, there exists a system of fundamental real units with the following property:

$$
\eta_{i}^{s-r^{2 i}}=\left(\eta^{\prime}\right)_{i}{ }^{l}, \quad(i=1,2, \ldots,(l-3) / 2),
$$

using the Kronecker-Hilbert symbolic powers. In the present note it is shown that certain units $\gamma_{i}, i=1,2, \cdots,(l-3) / 2$, which are analogous to those defined by Hilbert (Bericht, p. 432), may be expressed in forms involving the $\eta$ 's. This paper will appear in the Annals of Mathematics.

28. Professor H. S. Vandiver: Some theorems concerning properly irregular cyclotomic fields.

This article contains a number of theorems concerning properly irregular cyclotomic fields. Statements of these theorems with proofs indicated appeared in Proceedings of the National Academy of Sciences, vol. 15 (1929), p. 202-207. Full proofs will appear later in the Transactions of this Society.

29. Professor H. S. Vandiver: Summary of results and proofs on Fermat's last theorem. Fourth Paper.

This note contains a number of results concerning Fermat's last theorem. Statements of the theorems without proofs appeared in Proceedings of the National Academy of Sciences, vol. 15 (1929), pp. 108-109. The proofs of the results will appear in the Transactions of this Society.

30. Professor Lennie P. Copeland: Matrix conditions that the ternary cubic locus may have multiple points.

It is the purpose of the present paper first to determine a minimum set of necessary and sufficient conditions that the general ternary cubic locus (degenerate) may have exactly two double points, and second to show that there is a locus analogous to the ternary Hessian in the case of the degenerate polar cubic, since the locus of points whose polar cubic with respect to a given quartic break up into three straight lines is expressible as the three necessary and sufficient conditions that a cubic be factorable into linear factors.

31. Professor C. G. Latimer: On the class number of the cubic cyclotomic field.

In a former paper, the writer determined the prime ideals in the cubic Galois, or cyclotomic, field. Employing this result, the author determines the class number of the field in terms of the regulator. The method is similar to the well known method of treating the same problem for the quadratic field.

32. Professor G. W. Smith: Some results obtained by generalizing the analytical definition of a conic.

The ordinary analytical definition of a conic is generalized by considering the eccentricity as a function of either or both of the coordinates of the generating point. Some general properties of the loci whose equations are 
thus determined are given. Certain special functions for the eccentricity lead to interesting results in both rectangular and polar coordinates. It is shown that any curve whose equation is $f(x, y)=0$ may be considered as having a directrix and a focus, the eccentricity being variable.

33. Mr. R. G. Smith and Professor E. B. Stouffer: $A$ proof of a theorem of Halphen and its extension to $n$ dimensions.

If two curves in ordinary space have contact of order $m$ at a point, there exists a unique plane from any point of which one can project the two curves by means of two cones with contact of order $m+1$. This theorem was proved by Halphen in 1880 (Journal de l'Ecole Polytechnique, vol. 47 , p. 25). In the present paper a simpler proof of the same theorem is given, and the theorem is also extended to curves in contact in space of $n$ dimensions.

34. Dr. Marie J. Weiss (National Research Fellow): The limit of transitivity of a substitution group.

In this paper inequalities giving the relation between $n$, the degree of a $t$-ply transitive group, and $t$, the transitivity of the group, are found. The following theorems are proved: (1) Let the order of the subgroup $G_{t}$ that fixes $t$ letters of a $t$-ply transitive group of degree $n$ be divisible by an odd prime. Then if $t \geqq 8,(n-2 t+1) / 2 \geqq t ! /\{\delta !(t-\delta) !\}$, where $\delta$ is the greatest integer less than the quantity $t-(t-k+1)(\log 2) /(k+\log 2), k$ an integer such that $5 \leqq k \leqq t-1$; or $(n-2 t+1) / 2 \geqq t 2^{\alpha}, \alpha$ an integer $\geqq 4$ and $\geqq k-3$ $-\log k / \log 2$; or $(n-2 t+1) / 2 \geqq 2^{\gamma}, \gamma$ an integer $\geqq 4$ and $\geqq t-3-\log t / \log 2$. (2) Let $G_{t}$ be of order $2^{\mu}$. Then if $t \geqq 8,(n-t) / 2 \geqq t ! /\{\beta !(t-\beta) !\}$, where $\beta$ is an integer chosen such that $5 \leqq \beta \leqq t-3$, or chosen to be one unit less than the least value of $\beta$ which satisfies the inequality

$$
\begin{gathered}
{[(\beta+1) / 2] ! \geqq 2 t ! /\{\beta !(t-\beta) !\} ;} \\
\text { or } n-t \geqq[(t+1) / 2] ! ; \text { or } n-t \geqq t ! /[t / 2] !^{2} .
\end{gathered}
$$

The symbol $[s]$ denotes the integral value of $s$.

\section{Dr. L. W. Griffiths: A theorem on extended polygonal} numbers.

If $m$ is a positive integer, and $x=1,0,-1,-2,-3, \cdots$, the integers $e(x)=x+m\left(x^{2}-x\right) / 2$ are all the extended polygonal numbers of order $m+2$ and the first polygonal number, $e(1)=1$. Dickson has proved that every positive integer is the sum of $m$ values of $e(x)$ if $m \geqq 6$, but of $m+1$ values if $m=3,4,5$. A generalization of this theorem is suggested by the following restatement. Let $n, a_{1}, \cdots, a_{n}$ be positive integers, and $e_{1}, \cdots, e_{n}$ be values of $e(x)$; then the function $f=a_{1} e_{1}+\cdots+a_{n} e_{n}$ is universal when $n=m \geqq 6$, or $n=m+1$ if $m=3,4,5$, and $a_{1}=\cdots=a_{n}=1$. In $+a_{n} \leqq m$, and also for $m=3,4,5$, and $a_{1}+\cdots+a_{n} \leqq m+1$. For $m=1,2$ the problem reduces to that of polygonal numbers.

36. Professor L. S. Hill: Concerning linear transformations whose coefficients are matrices. 
The term "ring" being used as specified in Hasse, Höhere Algebra, part 1, pp. 7-9, any finite or infinite ring containing a unit element (which is necessarily unique) is here called a "scale." In particular, every field, and every "Integritätsbereich," is a scale. The set of all square matrices, of any assigned order, in which the elements are elements of the scale $S$ is called a "range," $\Gamma$. The ordinary type of matrix algebra is set up in $\Gamma$, and attention is directed to the transformation

$T \quad y_{1}=a_{11} x_{1}+\cdots+a_{1 n} x+a_{1}, \cdots, y_{n}=a_{n 1} x+\cdots+a_{n n} x_{n}+a_{n}$,

defined by $n$ linear equations in which the $x_{i}, y_{i}, a_{i j}, a_{i}$ denote matrices of $\Gamma$. Conditions for the existence of a unique inverse transformation $T^{-1}$, and means to determine its equations, are given. A facile construction is presented for transformations $T$, of any desired complexity, which are involutory (that is, of period 2). All results are valid in ranges of matrices whose elements are in turn matrices, the elements of these elements being perhaps again matrices, etc. An application is made in the preparation of cipher transformations offering extraordinary resistance to cryptanalysis.

37. Professor A. D. Campbell: Note on linear transformations of $n$-ics in $m$ variables.

This paper has appeared in the September-October number of this Bulletin.

38. Mr. D. H. Lehmer: Numerical integration as applied to Dirichlet series.

In the literature on the analytic theory of numbers, there are a large number of "identities" between certain Dirichlet series $\sum_{n=1}^{\infty} a_{n} n^{-8}$ and simple expressions involving Riemann's $\zeta$ function. The methods used in proving these identities vary considerably; for the most part, however, use is made of the infinite product definition of $\zeta(s)$. The purpose of this note is to indicate a principle of unification by means of which all these identities fall into classes such that all identities of the same class may be obtained simultaneously. This unifying principle is based on Bougaieff's concept of numerical integration of a function $f(n)$ defined by the relation $F(n)=\sum_{\delta / n} f(\delta)$. We consider a sequence $f_{r}(n)$ defined for all positive and negative integer values of $r$ by the relation $f_{r+1}(n)=\sum_{\delta / n} f_{r}(\delta)$, the fundamental function $f_{0}(n)$ being specified. In this way the function $f_{0}(n)$ defines a class of Dirichlet series $\sum_{n=1}^{\infty} f_{r}(n) n^{-s}$. Most of the known identities belong to three classes defined by $f_{0}(n)=n^{k}, \log n, \lambda(n)$, the latter being Liouville's function. By introducing new functions $f_{0}$ one obtains the rest of the known identities as well as many new ones.

39. Professor Raymond Garver: Quartic equations with the alternating group.

Baker and Seidelmann have given expressions for the most general quartic equation whose Galois group is the alternating group. The present paper shows that Baker's form actually does not represent all such quartics, while Seidelmanns' form does. The verification of this last fact 
is entirely independent of Seidelmann's work, and requires only rather elementary algebraic manipulations.

40. Professor Raymond Garver: Transformations on cubic equations.

This paper, which will be published in the American Mathematical Monthly, studies exceptional Tschirnhaus transformations on cubic equations which are not equivalent to linear fractional transformations. A number of statements in the literature deny the existence of such transformations, and they are perhaps worth mentioning on that account as well as because they lead to certain rather interesting results.

41. Professor W. E. Roth: On the unilateral equation in matrices.

Let $A_{i}, i=0,1, \cdots, p$, be rectangular matrices of $m$ rows and $n$ columns and let $X$ be an unknown square matrix of order $n$; then the equation $\sum_{i=0}^{p} A_{i} X^{p-i}=0$ is said to be unilateral (Sylvester, Philosophical Magazine, vol. 17 (1884), p. 392). The present paper develops the necessary conditions for the existence of $X$ satisfying this equation and in particular extends a theorem due to Sylvester regarding the latent (characteristic) roots of the unknown matrix (Comptes Rendus, vol. 99 (1884), pp. 621-631). This theorem for our more general equation may be stated thus: If $\sum_{i=0}^{p} A_{i} \lambda^{p-i}$ is the matrix obtained from the left member of the equation above when $X$ is replaced by the scalar variable $\lambda$, then the latent roots of $X$ must be values of $\lambda$ for which the rank of $\sum_{i=0}^{p} A_{i} \lambda^{p-i}$ is less than $n$. The converse is not true. The results apply equally well when the unknown matrix of order $m$ occurs on the left of all terms of the given equation.

42. Mr. John McDonnell: Note on Fermat's last theorem.

The author proves that if $x^{p}+y^{p}+z^{p}=0$, then (1) if $y z+z x+x y \neq 0$ $(\bmod p)$, and $r$ be any factor of $x^{2}-y^{2}$, then $r^{p-1} \equiv 1\left(\bmod p^{2}\right)$, and $(2)$ if $x(y-z)\left(x^{2}+y^{2}\right) \neq 0(\bmod p)$, and $r$ be any factor of $x^{2}+y z$, then $r^{p-1} \equiv 1$ $\left(\bmod p^{2}\right)$. The proof resembles a proof of Furtwängler's for a similas result.

43. Professor H. A. Simmons: Maximum numbers associated with a certain diophantine equation.

This paper presents for the equation

$$
\sum\left(1 /\left(x_{1} x_{2} \cdots x_{n-1}\right)\right)=b /[(m+1) b-1]
$$

a solution in positive integers which has three special properties, namely: it contains the largest number $x$ that can appear in any solution in which $x_{1}, x_{2}, \cdots, x_{n-1}$ are positive integers, with $x_{n}$ only restricted to be a real number $\geqq x_{i}, i=1,2, \cdots, n-1$; and, with the same hypotheses on the $x$ 's, the solution found maximizes the sum $x_{1}+x_{2}+\cdots+x_{n}$ and the product $x_{1} x_{2} \cdots x_{n}$. The final section of the paper exhibits for the equation $\sum\left(1 /\left(x_{1} x_{2} \cdots x_{r}\right)\right)=b /[(m+1) b-1], r$ being less than $n$, the 
number of variables in the symmetric function, a solution in positive integers which includes as a special case the solution whose properties were mentioned above.

\section{Mr. J. H. Roberts: A non-dense continuum.}

The author has shown that if $M$ is a square plus its interior then there exists an upper semi-continuous collection $G$ of mutually exclusive continua such that (1) every point of $M$ belongs to some continuum of $G$, (2) $G$ is topologically equivalent to $M$, and (3) each continuum of $G$ contains more than one point. This question arises: "Is it necessary that a given continuum $M$ contain a domain in order that such a set $G$ exist?" In the present paper an example is given which shows that the answer to the above question is in the negative.

\section{Dr. Leo Zippin: Continuous curves and the Jordan curve theorem.}

It is shown that if a continuous curve $C$, is such that (1) every simple closed curve of $C$ disconnects $C$, and (2) no arc of $C$ disconnects $C$, then $C$ satisfies the Jordan Curve-Theorem and is homeomorphic with the complement on a simple closed surface of a closed and totally disconnected point set (possibly vacuous, if $C$ is compact); these surfaces the author has previously termed cylinder-trees. It is proved that a necessary and sufficient condition that a continuous curve $C$ containing at least one simple closed curve be a cylinder-tree, is that if $K$ is any simple closed curve of $C$, then $K \subset \overline{\mathrm{C}-\mathrm{K}}$ and $C-K$ is the sum of two components. The author shows that the following postulate-set characterizes the number-plane: Axioms 1-5 of R. L. Moore's $\Sigma-1$, and Axiom $8^{\prime}:$ if $K$ is a simple closed curve of the space $S$, then $K \subset \overline{S-K}$, and $S-K$ is the sum of two components at least one of which is compact. Further, if in this system Axiom 2 be replaced by Axiom $\eta^{\prime}$ : a region is a connected set of points not a single point, Axiom 3 may be replaced by $3^{\prime}:$ if $x$ is a point of $S, S-x$ is connected, or else by $3^{\prime \prime}:$ if $m$ is an $\operatorname{arc}$ of $S$, there is a point $x$ of $m$ such that $S-x$ is connected.

\section{Professor G.T.Whyburn: Concerning continuous curves} and denodular sets.

A connected and locally connected set is denodular if all its nodular elements (that is, elements corresponding to the cyclic elements of a continuous curve) are points. It has previously been shown by the author that a denodular set is highly similar to a connected subset of an acyclic continuous curve. In this paper a study is made of the conditions under which a continuous curve may be expressed as the sum of a denodular set and another set $H$ of simple structure. For example, $H$ may be finite or may be null-dimensional. In this way characterizations of various types of continuous curves are obtained. For example, it is shown that a continuous curve is one-dimensional if and only if it is the sum of a denodular set and a null-dimensional set. 
47. Professor R. L. Wilder: Extension of a theorem of Mazurkiewicz.

Mazurkiewicz has recently shown (Fundamenta Mathematicae, vol. 13, pp. 146-150), in answer to a question proposed by Knaster, that if $A$ is a closed set in $E_{n}$ which is homeomorphic with a subset of $E_{n-1}$, then all points of $A$ are accessible from $E_{n}-A$. It is shown in the present paper that the points of $A$ are regularly accessible from $E_{n}-A$. In the proof the following fundamental lemmas are established: (1) If $a$ is a point of $A$, and $S$ is a spherical neighborhood of $a$, then if the component $C$ of $S \cdot A$ which contains $a$ separates $S$, the set $C$ is homeomorphic with a connected open subset of $E_{n-1}$. (2) If $A$ separates two points $P$ and $Q$ in some spherical neighborhood $S$, there exists a component of $A \cdot S$ which separates $P$ and $Q$ in $S$.

48. Professor R. L. Wilder: Concerning perfect continuous curves.

The following results hold in any locally compact metric space: (1) Every compact connected subset of a perfect continuous curve has property $S$. (2) Every component of a quasi-open subset of a perfect continuous curve is arcwise connected im kleinen. (3) In order that a continuum $M$ should be a perfect continuous curve, it is necessary and sufficient that the boundary points of the components of quasi-open subsets of $M$ should be regularly accessible. (4) If $K$ is a closed and bounded set lying in a component $Q$ of a quasi-open subset of a perfect continuous curve, then $K$ is a subset of a continuous curve which itself lies in $Q$.

49. Professor R. L. Wilder: A converse of the Jordan. Brouwer separation theorem in three dimensions.

This paper presents a converse, in $E_{3}$, of the Jordan-Brouwer separation theorem. (See J. R. Kline, this Bulletin, vol. 34, pp. 156-159.) The result may be stated as follows: Let $K$ be a closed and bounded set in $E_{3}$ such that (1) the Betti numbers $(\bmod 2) R^{0}\left(E_{3}-K\right)$ and $R^{1}\left(E_{3}-K\right)$ are respectively equal to 2 and $1,(2)$ if $D$ is a component of $E_{3}-K$, the 0 -chains in $D$ are uniformly homologous to zero in $D^{*}$, and every point of $K$ is a limit point of $D$. Then $K$ is a simple closed surface. Another statement of the result, not limiting the number of complementary domains, but strengthening the accessibility conditions, is also given.

50. Dr. W. L. Ayres (National Research Fellow): On the density of the cut points and end points of a continuous curve.

* That is, for any number $e>0$, there exists a number $d$ such that if $L^{0}$ is a 0 -chain of $D$ of diameter less than $d, L^{0}$ bounds a chain $L^{1}$ in $D$ of diameter less than $e$. This property is equivalent to uniform connectedness im kleinen here, and does not imply that a set is connected. 
The author proves the following relations between the density of the end points and that of the cut points of a continuous curve $M$ : the set $K$ of all cut points of $M$ is dense in $M$ if, and only if, the end points of $M$ are dense in $M-K$. In order that the end points of $M$ be dense in $M$ it is necessary and sufficient that the set of all cut points of Menger-Urysohn order $\geqq 3$ be dense in $M$. When the end points are dense in $M$, the set of all cut points of order 2 is zero-dimensional. And conversely, when the set of cut points of order 2 is zero-dimensional and the set of all cut points is dense in $M$, then the end points are dense in $M$.

51. Dr. W. L. Ayres: On joining subsets of a continuous curve by arcs and simple closed curves.

It is shown that if $K$ is a finite subset of $m$ points of a continuous curve $M$ and no two points of $K$ may be separated in $M$ by a set of $m-1$ points of $M$, then there is a simple closed curve of $M$ containing $K$. If no two points of $K$ are separated by $m-2$ points, there is an arc of $M$ containing $K$. Examples are given to show that the numbers $m-1$ and $m-2$ cannot be reduced, but for the special case of a plane continuous curve $M$ the following sharper theorem is true: If $M$ remains connected on the omission of any pair of points, then every finite subset lies on a simple closed curve of $M$.

52. Dr. W. L. Ayres: On continuous images of a compact metric space.

In this note it is shown that if $M$ is a set of points and for each sequence $\left[q_{i}\right]$ of $M$ there exists a "limit" function such that (1) limit $\left[q_{i}\right]$ is a unique subset of $M$ (which may be vacuous), (2) if limit $\left[q_{i}\right] \neq 0$ and $\left[q_{n_{i}}\right]$ is a subsequence of $\left[q_{i}\right]$ then limit $\left[q_{n_{i}}\right]=\operatorname{limit}\left[q_{i}\right]$, and further (3) if $M$ is the continuous image of a compact metric space, then $M$ is compact and metric. The result is a generalization of a theorem of Alexandroff (Mathematische Annalen, vol. 96, p. 562).

53. Professor W. A. Wilson: A property of continua equivalent to local connectivity.

This note proves the following theorem: If $M$ is a metric space which is compact, separable, and connected, the necessary and sufficient condition for $M$ to be locally connected at every point is that, for every pair of subcontinua $A$ and $B$ of $M$, there is a decomposition of $M$ into two continua $P$ and $Q$ such that $P \cdot B=Q \cdot A=0$. The same conclusion is valid if $M$ is a bounded or unbounded continuum lying in a euclidean space.

54. Professor L. L. Dines: Independent postulates for a generalized euclidean algorithm.

In a Königsberg dissertation Plaut developed an algorithm for finding the highest common factor of a finite set of expressions of a general nature, including algebraic polynomials and linear differential and difference expressions as special cases. He based the development upon twelve postu- 
lates, the mutual independence of which he said he had been unable to establish. In the present note it is shown that these postulates are not independent, and an equivalent set of ten independent postulates is presented.

\section{Professor A. J. Kempner: On the roots of algebraic} equations and derived equations.

This paper contains miscellaneous results of the following character: (1) A uniform method of derivation of a large number of known theorems such as If $f=0$ (real coefficients) has all roots real and distinct, then $f-k f^{\prime}=0$ has only real roots; $f^{\prime} \cdot f^{\prime}-f \cdot f^{\prime \prime}=0$ has only complex roots; $f^{2}+k^{2} f^{\prime 2}=0$ has only complex roots; $f^{2}-k^{2} f^{\prime 2}=0$ has only real roots, and new results of related type. (2) For the Legendre polynomials $P_{n}$ the equation $P_{n}{ }^{\prime} \cdot P_{n}{ }^{\prime}$ $-P_{n} \cdot P_{n}{ }^{\prime \prime}=0$ has all roots imaginary and of absolute value $<1$. A general theorem is given concerning the location of all roots of $f^{\prime} \cdot f^{\prime}-f \cdot f^{\prime \prime}=0, f$ a polynomial. (3) A more precise form of Jensen's theorem concerning roots of $f^{\prime}=0, f$ a polynomial with real coefficients.

56. Professor H. E. Buchanan: On a certain function of the masses in the three-body problem.

In a recent paper (American Journal, vol. 50 (1928), No. 4) on the three-body problem, the writer encountered the following function of the masses: $f\left(m_{1}, m_{2}, m_{3}\right) \equiv\left(m_{1}+m_{2}+m_{3}\right)^{2}-27\left(m_{1} m_{2}+m_{1} m_{3}+m_{2} m_{3}\right)$. The present paper discusses this function in detail. If $f\left(m_{1}, m_{2}, m_{3}\right)>0$ the solutions of the differential equations for small oscillations contain only periodic terms and terms linear in $t$. On the other hand if $f\left(m_{1}, m_{2}, m_{3}\right) \leqq 0$, the positions are unstable due to the presence of terms of the form $e^{a t}$ as well as terms linear in $t$. If Gyldén (Bulletin Astronomique, vol. 1) and Moulton (Astronomical Journal, No. 483) are correct in their explanation of the Gegenschein it seems that there ought to be a much larger accumulation of matter near the equilateral triangle points than near the straight line positions, for the latter positions are always unstable with the exponential terms present. This paper has been accepted by the American Journal and will appear in an early number.

\section{Professor H. L. Olson: Doubly divisible quaternions.}

This paper derives a necessary and sufficient condition that a rational integral quaternion $a$ be both a left and a right factor of a rational integral quaternion $x$.

58. Mr. J. R. Carson: The asymptotic solution of an operational equation.

This paper deals with the asymptotic solution of a specific operational equation with particular reference to the order of magnitude of the error committed by stopping with the numerically smallest term of the series, and the effect on numerical accuracy of adding an additional exponential term. The paper closes with a brief discussion of the general operational equation 
and the conditions under which its divergent solution, where such exists, conforms to Poincaré's definition of an asymptotic series.

\section{Professor C. H. Rowe: A kinematical treatment of some theorems on normal rectilinear congruences.}

The author considers a two-parameter motion of a straight line $l$ and a two-parameter motion of a rectangular trihedral, or observer, $T$, which is in one-to-one correspondence with that of $l$. He studies the relationship between the actual congruence and the apparent congruence which $l$ describes, that is the congruence actually described by $l$ and the congruence which $l$ appears to describe to the observer $T$, and establishes a sufficient condition that the actual congruence be normal when the apparent congruence is normal. By means of this condition, he proves a number of known theorems and obtains extensions of certain of them.

\section{Professor R. G. Lubben: Concerning limiting sets.}

In this paper the following theorems are proved: (1) In a space $L$ which has the distributive property, every uncountable point set has a limit point. (2) In a space $S$ which has the distributive property, every uncountable point set contains a limit point of itself. (3) In order that a metric space have the distributive property, it is necessary and sufficient that it be locally compact and separable.

R. G. D. RichaRdson,

Secretary 